\title{
Evaluation of Life Quality and Dyspnea Intensity on Chronic Airway Diseases Patients in Pulmonary Rehabilitation Program
}

\author{
Kathleen Julia Silva', Giseli Domingues Cordeiro' ${ }^{1}$, Caroline Rossinoli², \\ Maurício Longo Galhardo 3 , Márcia Maria Faganello ${ }^{4}$ \\ ${ }^{1}$ Academic of Department of Physiotherapy, Unisalesino, Lins, Brazil \\ ${ }^{2}$ Department of Physiotherapy, Hospital Unimed, Lins, Brazil \\ ${ }^{3}$ Department of Pneumonology Unimed, Lins, Brazil \\ ${ }^{4}$ Department of Physiotherapy, UNESP, Marilia, Brazil \\ Email: marciafaganello@gmail.com
}

Received 26 October 2015; accepted 22 December 2015; published 25 December 2015

Copyright (C) 2016 by authors and Scientific Research Publishing Inc.

This work is licensed under the Creative Commons Attribution International License (CC BY). http://creativecommons.org/licenses/by/4.0/

c) (7) Open Access

\section{Abstract}

The chronic obstructive pulmonary disease (COPD) and asthma are chronic airway diseases that cause considerable physical, emotional and social restrictions. The life quality of patients who suffer from these diseases, is more affected by dyspnea then by other symptoms. That way is possible to correlate the impact of dyspnea on their life quality. The Pulmonary Rehabilitation Program (PRP) helps to improve the physical fitness and quality of life. This paper presents a study of the life quality and the dyspnea intensity in chronic lung disease patients. The research has participation of fourteen patients, distributed as seven suffering from COPD and the other seven suffering from asthma, for both gender and with an age average of 74.2 \pm 8.9. The patients answer the following questionnaires: Baseline Dyspnea Index (BDI), Medical Research Council (MRC) Dyspnea Scale, Airways Questionnaire 20 (AQ20) and the Brazilian version of the ShortForm (SF-36) life quality questionnaire. From the present study, it was concluded that asthma patients have a less impaired life quality, since the dyspnea intensity is lesser, compared to the one with COPD.

\section{Keywords}

Life Quality, Dyspnea Intensity, Chronic Lung Disease 


\section{Introduction}

The terminology used to describe the difficulty to breathe, on certain patients having some pulmonary, cardiac or metabolic disease, is dyspnea. And the obstruction, inflammation and hyper responsiveness of airways, developed by allergens, environmental exposures and genetic factor, are characteristics of an inflammatory disease called asthma. They are classified according to their frequency, intensity of their symptoms, exercise tolerance, number of hospitalization, medication and have already made use of mechanical ventilation. They can be classified as intermittent, mild persistent, moderate persistent and severe persistent.

According [1], in 2004, the Chronic Obstructive Pulmonary Disease (COPD) occupied the 13th place in the world ranking of conditions, however it is estimated that by 2030 COPD will occupy the 5th place in the ranking. COPD is defined as a group of conditions characterized by the presence of obstruction or chronic airflow limitation slowly progressive, persistent and irreversible [2]. In COPD is a combination of Pulmonary Emphysema and Chronic Bronchitis. When there is change of the lung parenchyma, resulting in an increase of the airspaces distal to the terminal bronchioles, with destruction of their walls, we have emphysema. If the chronic inflammatory process causes bronchial changes, is called Chronic Bronchitis. In 2004, an estimated 64 million people were living with COPD worldwide, more than 3 million people died of COPD in 2005 equaling 5\% of all deaths in the world that year [3].

In relation to health, quality of life (QoL) is a component that is a perception indicator that the patient has the disease in their welfare. According [4], the loss of physical capacity and QoL may also be related to the patient's psychic changes. The physiological and functional limitations of physical ability are accompanied by psychological, and social isolation resulting in deterioration of quality of life.

The questionnaires were developed in order to assess the health status and the effects, which cause disease, being an instrument, used to compare the effect of an intervention [5]. For Pulmonary Rehabilitation, these instruments should be short and easy to understand, self-reported or not, and own property to distinguish between those who have chronic lung disease better or worse QoL [6].

This paper is due the following question: "Patients with chronic lung disease have impaired quality of life because of the degree of dyspnea this?”. Against the various prerogatives addressed, this work has as main objective to evaluate the quality and intensity of dyspnea in chronic lung disease.

\section{Pulmonary Rehabilitation}

Pulmonary Rehabilitation was defined in 2006 by the ATS (American Thoracic Society), as a multidisciplinary approach based on evidence of symptomatic patients with chronic respiratory diseases and often has reduced their activities of daily living. Pulmonary Rehabilitation aims to: reduce airway obstruction, in order to alleviate and control the symptoms and pathophysiology of respiratory failure; panic desensitize the patient's dyspnea; improve their nutritional status; introduce exercise into the lives of patients stopping the vicious cycle of inactivity, and teach the techniques and strategies for energy conservation.

Physical activity is beneficial to many of the structural and functional components of the musculoskeletal system, increasing functional capacity, improving QoL [7]. Pulmonary Rehabilitation is an important resource to be used, associated with therapeutic intervention and cessation of smoking. Integrates the maintenance of clinical stability of chronic lung disease [6].

Patients attending in Pulmonary Rehabilitation Program (PRP) improve QOL and exercise capacity, reduce the need for home medical visits when exacerbations occur, and reduce days of hospitalization.

\section{Development}

\subsection{Materials and Methods}

The study included 14 adult patients of both genders, and 7 patients with COPD and 7 patients with asthma, participants in PRP. The study period was from March to September 2012.

Patients were include by criteria for COPD according to the guidelines established in the Global Initiative for Chronic Obstructive Lung Disease with a post bronchodilator forced expiratory volume in the first second (FEV1)/forced vital capacity (FVC) ratio, 0.70 and an increase in FEV1 of, 15\% or $200 \mathrm{~mL}$ after inhalation of a $\beta 2$-agonist [8].

The diagnosis of asthma is confirmed by the presence of airflow obstruction characterized by FEV1less than 
$80 \%$ of predicted and FEV1/FVC ratio (below 75\%) that improves significantly after bronchodilator (increase in FEV1 of 7\% over the value planned and $200 \mathrm{ml}$ in absolute terms, after inhaled beta-2 agonist short) [9].

This study was conducted in the Athletic Association of Brazil-Bank AABB located in Linscity in the period from 7:30 am to 9 am, when we analyzed the intensity of dyspnea and quality of life.

The 14 patients were analyzed by the Brazilian version of the Quality of Life questionnaires Short Form-36 (SF-36) questionnaires and Airways Questionnaire 20 (AQ20). To evaluate the intensity of dyspnea were applied the Basal dyspnea index (BDI) and dyspnea scale of the Medical Research Council (MRC).

The Brazilian version of the questionnaire Quality of Life Short Form-36 (SF-36) consists of 36 questions, divided into eight domains: physical functioning, bodily pain, general health, vitality, social functioning, emotional and mental health. The data are evaluated, from the transformation of the response scores from 0 to 100 , of each component, there is no single value that summarizes the entire assessment, revealing in a general health better or worse.

Airways Questionnaire 20 (AQ20) aims to evaluate the quality of life of patients with respiratory diseases obstructive character. With the response options "yes", "no" and "not applicable", the end result varies from 0 to 20 points. Higher scores indicate poorer quality of life.

The basal level of dyspnea (BDI) is composed of three items, which are intended to assess the degree of disability caused by the impact of dyspnea The final score ranges from 0 to 12; the highest score is higher performance as the symptom.

The scale of dyspnea Medical Research Council (MRC), designed to analyze the intensity of dyspnea in the patient as he performs some kind of task in your daily life, your score is 0 to 5 . The higher the score, the greater is the sensation of dyspnea.

After collecting these data, statistical analysis was performed and the values were shown in tables. For the statistical analysis the Spearman correlation was used for parametric variables not.

The small sample number of participants is due to the fact of non-adherence of patients to the rehabilitation program.

\subsection{Results}

We selected 18 patients of both genders participants PRP. During the study were excluded two individuals for presenting interstitial lung disease, and 2 for not attending the PRP, thus 14 patients. The BDI and MRC questionnaires were applied to verify the intensity of dyspnea, and the AQ20 and the SF-36 to assess the quality of life in the period from March to September. Characteristics of the COPD patients and Asthma are showed in the Table 1.

Table 1. Characteristics of patients with COPD and Asthma.

\begin{tabular}{cccc}
\hline & Total $(\mathrm{n}=14)$ & COPD $(\mathrm{n}=7)$ & Asthma $(\mathrm{n}=7)$ \\
\hline Age (years) & $74.2 \pm 8.9$ & $76.7 \pm 10.3$ & $71.2 \pm 6.8$ \\
BDI & $7.9 \pm 3.0$ & $7.3 \pm 3.9$ & $8.6 \pm 1.9$ \\
MRC & $1.6 \pm 0.8$ & $1.8 \pm 0.9$ & $1.4 \pm 0.8$ \\
AQ20 (\%) & $25.8 \pm 20.4$ & $37.5 \pm 22.1$ & $15.7 \pm 13.0$ \\
SF36-FC & $76.9 \pm 18.2$ & $63.3 \pm 17.5$ & $88.6 \pm 8.0$ \\
SF36-PAL & $55.8 \pm 41.0$ & $29.2 \pm 24.6$ & $78.6 \pm 39.3$ \\
SF36-Pain & $70.2 \pm 26.8$ & $77.3 \pm 28.2$ & $64.1 \pm 26.0$ \\
SF36-GH & $83.0 \pm 11.3$ & $75.0 \pm 8.1$ & $90.0 \pm 8.8$ \\
SF36-V & $59.3 \pm 18.3$ & $56.8 \pm 26.8$ & $61.4 \pm 8.0$ \\
SF36-SA & $97.5 \pm 7.9$ & $91.7 \pm 14.4$ & $100.0 \pm 0.0$ \\
SF36-EA & $90.0 \pm 31.6$ & $75.0 \pm 50.0$ & $100.0 \pm 0.0$ \\
SF36-MH & $80.6 \pm 8.9$ & $77.3 \pm 8.3$ & $83.4 \pm 9.1$ \\
\hline
\end{tabular}

$\mathrm{BDI}=$ baseline dyspnea index; $\mathrm{MRC}=$ scale of the Medical Research Council; AQ20 = process of questionnaire flight 20; Short-form; SF36-FC = Short-form functional capacity; SF36-PAL = Short-form physical aspects limitation; SF36-Pain = Short-form pain; SF36-GH = Short-form general health; SF36-V = Short-form vitality; SF36-SA =Short-form social aspect; SF36-EA = Short-form emotional aspects; SF36-MH = Short-form mental health. 
Table 1 shows demographic data and age and the values of BDI questionnaires, AQ20, SF-36.

Table 2 shows the comparison of QoL questionnaires by AQ20 and the SF-36.

According to values shown in Table 2, we can demonstrate that patients with COPD are more impaired QoL compared to patients with asthma. These data were found in the values of AQ20 and domains (CF, LAF and EGS) of the SF-36. The analysis of other areas was not statistically significant.

Table 3 shows dyspnea intensity of interference in the quality of life in patients with COPD and Asthma.

In the analysis of dyspnea intensity evaluated by BDI and MRC, we find that the greater the intensity of dyspnea is more impaired the quality of life of patients with asthma and COPD.

\section{Discussion}

The importance of this study was to analyze the intensity of dyspnea, through questionnaires BDI, MRC and QoL with the SF-36 and the AQ20. In cases of chronic lung disease COPD and Asthma several factors such as dyspnea and physical deconditioning may interfere with activities of daily living (ADL) and the individual's quality of life [10] (FERREIRA, GREEN, ROCHA, 2009).

Quality of life has been extensively studied by means of quality of life questionnaires, however there are few studies in Latin America that have evaluated this aspect in depth [11] [12].

In our study, to analyze the degree of bronchial obstruction with AQ20, concluded that, especially in patients with COPD, as dyspnea intensity increases, QOL is more impaired. For [6], dyspnea is the symptom that most physically limited individuals with COPD.

Patients with chronic airflow limitation present limitation of physical capacity due to abnormality of the ventilatory mechanisms, the respiratory muscles, the alveolar gas exchange and cardiovascular function [13].

In our study, individuals with COPD were negatively correlated with the MRC LAF areas EGS questionnaire SF-36. So it can be seen that individuals with COPD showed a higher involvement of QOL compared with

Table 2. Evaluation of the quality of life.

\begin{tabular}{rccc}
\hline & COPD $(\mathrm{n}=7)$ & Asthma $(\mathrm{n}=7)$ & $\mathrm{p}$ \\
AQ20 & $37.5 \pm 22.1$ & $15.7 \pm 13.0$ & $0.049^{*}$ \\
SF36-FC & $63.3 \pm 15.5$ & $88.6 \pm 8.0$ & $0.006^{*}$ \\
SF36-PAL & $37.5 \pm 0.0$ & $100.0 \pm 62.5$ & $0.035^{*}$ \\
SF36-GH & $75.0 \pm 8.1$ & $90.0 \pm 8.8$ & $0.009^{*}$ \\
\hline
\end{tabular}

AQ20 = process of questionnaire flight 20; SF36-FC = Short-form functional capacity; SF36-PAL = Short-form physical aspects limitation; SF36-GH $=$ Short-form general health; ${ }^{*} \mathrm{p}<0.05$.

Table 3. Dyspnea intensity of interference in the quality of life in patients with COPD and Asthma.

\begin{tabular}{ccccc}
\hline SF-36 domains & Correlation with BDI & $\mathrm{p}$ & Correlation with MRC & $\mathrm{p}$ \\
\hline SF36-FC & 0.34 & 0.244 & -0.33 & 0.252 \\
SF36-PAL & 0.23 & 0.435 & -0.75 & $0.002^{*}$ \\
SF36-Pain & -0.34 & 0.236 & -0.11 & 0.723 \\
SF36-GH & 0.15 & 0.616 & -0.61 & $0.025^{*}$ \\
SF36-V & -0.22 & 0.447 & -0.19 & 0.516 \\
SF36-SA & -0.47 & 0.148 & -0.26 & 0.446 \\
SF36-EA & 0.29 & 0.384 & -0.19 & 0.583 \\
SF36-MH & 0.04 & 0.878 & -0.00 & 0.978 \\
AQ20 & -0.71 & $0.006^{*}$ & 0.49 & 0.087 \\
\hline
\end{tabular}

AQ20 = process of questionnaire flight 20; SF36-FC = Short-form functional capacity; SF36-PAL = Short-form physical aspects limitation; SF36-Pain = Short-form pain; SF36-GH = Short-form general health; SF36-V = Short-form vitality; SF36-SA =Short-form social aspect; SF36-EA = Short-form emotional aspects; SF36-MH $=$ Short-form mental health. ${ }^{*} \mathrm{p}<0.05$. 
asthma patients.

The negative effects of asthma on QOL are related to the disease symptoms, side effects of medications or limitations at work or with exercise [14].

Studies by [15] showed that BDI/TDI (dyspnea Transition Index) indices are valid for use in clinical trials and have the ability to identify clinically important differences in dyspnea. In this study, the group of patients with COPD showed higher intensity of dyspnea in relation to the group of asthmatic patients, evaluated by BDI and MRC questionnaires.

Associates the progressive deconditioning physical inactivity the start to a vicious cycle in which a worsening of dyspnea is associated with smaller and smaller physical effort. These data confirm the negative correlation of the MRC, with the LAF areas, CF SF-36 [6].

For [16], exercise limitation is due to airflow limitation that generates mainly dyspnea during exercise. Thereby decreasing the daily activity can evolve into a sedentary lifestyle and social isolation, which can result in a high degree of anxiety and depression with worsening of QoL.

A study by Gonzalez et al. (2005) demonstrated the association between the intensity of dyspnea and health status. Regression analyzes show that the sensation of dyspnea explains between $25 \%$ and $54 \%$ of the variations in health status scores in patients with COPD. These data corroborate the results of this study in relation to EGS field.

Studies have shown that dyspnea constitutes the main limiting factor of QOL related to health of patients with chronic respiratory failure, either obstructive or restrictive nature [17].

The present study demonstrated that the group of COPD showed limitations in QoL due to the greater intensity of dyspnea compared to asthma group. Although, similar to the mechanism of airflow obstruction in COPD and asthma, and are functional distinct pathological features of the diseases. These differences may explain the better prognosis in patients with asthma compared with COPD (FABBRI et al. 2003).

Only three previous studies have examined the contribution of the sensation of dyspnea for AQ20 [12] [18] [19]. The AQ20 proved to be an instrument with discriminative power and responsiveness compared to other specific questionnaires [18].

By correlations was found that the functional capacity and the general health are relevant factors that may affect the quality of life for individuals with COPD participants PRP.

The results of this study corroborate the findings in the literature, demonstrating that asthma causes lower rate of dyspnea and improved QoL as the symptoms of COPD is progressive and worsens with age, while the symptoms of asthma are episodic and repetitive with time.

\section{Conclusions}

Through the analysis of the results obtained in the present study, we can conclude that the quality of life is directly influenced by the intensity of dyspnea in patients with COPD and Asthma.

By correlations with the domains, general health and physical aspects limitation (SF-36), patients with asthma had a lower dyspnea intensity index and better quality of life compared to patients with COPD. Direct interference of dyspnea in quality of life was also demonstrated in the analysis of BDI with the questionnaire AQ20.

We conclude that COPD is also a systemic disease, which causes more intense and hence more dyspnea impaired quality of life when compared with asthma.

\section{References}

[1] Ministry of Health (2012) Roflumilast for Chronic Obstructive Pulmonary Disease (COPD) Associated with Chronic Bronchitis.

[2] Pryor, J.A. and Webber, B.A. (2002) Physiotherapy for Respiratory Problems and Heart. Guanabara, Rio de Janeiro.

[3] World Health Organization (WHO) Chomic Obstructive Pulmonary Disease (COPD), 2011.

[4] Gava, M.V. and Picanço, P.S.A. (2007) Pulmonalogy Physiotherapy. Manole, São Paulo.

[5] Tarantino, A.B. (2002) Lung Disease. 5th Edition, Guanabara, Rio de Janeiro.

[6] Rodrigues, S.L. (2003) Pulmonary Rehabilitation: Basic Concepts. Manole, São Paulo.

[7] Allsen, P.E., Harrison, J.M. and Vance, B. (2001) Exercise and Life Quality: A Personalized Approach. 6th Edition, Manole, Barueri. 
[8] Global Initiative for Chronic Obstructive Lung Disease (2014) Global Strategy for the Diagnosis, Management, and Prevention of Chronic Obstructive Lung Disease-Updated 2014. Global Initiative for Chronic Obstructive Lung Disease, Vancouver. http://www.goldcopd.org/uploads/users/files/GOLD_Report2014_Feb07.pdf

[9] Global Initiative for Asthma-GINA (Homepage on the Internet). Global Initiative for Asthma, Bethesda. (Cited 2011 Apr 1) Global Strategy for Asthma Management and Prevention, 2010. (Adobe Acrobat Document, 119 p.) http://www.ginasthma.org/pdf/GINA_Report_2010.pdf

[10] Ferreira, E.H.R. (2007) Assessment of Life Quality and Attributes Related to Functional Capacity in Chronic Lung Disease. Salesian Catholic University Center-Auxilium, Lins, Monograph (Undergraduate Physiotherapy).

[11] Camelier, A.S. (2005) Life Quality Health Related Evaluation of COPD Patientes: A Population Based Study of the SF-12 in São Paulo-SP. São Paulo.

[12] Camelier, A.S. (2005) Airways Questionnaire 20 Brazilian Version: A Study and Correlations of Patients with COPD. American Journal of Respiratory and Critical Care Medicine, 99, 602-608. http://dx.doi.org/10.1016/j.rmed.2004.09.022

[13] Marcondes, M., Sustovich, D. and Ramos, O.C. (1979) Clinical Medical Workup and Pathophysiology. Guanabara Koogan Press, Rio de Janeiro.

[14] Scanlan, C.G., Wilkins, R.L. and Stoller, J.K. (2000) Fundamentals of Respiratory Therapy. Manole, São Paulo.

[15] Witek, J.T. and Mahler, D. (2003) Minimal Important Difference of the Transition Dyspnoea Index in a Multinational Clinical Trial. European Respiratory Review, 21, 267-272.

[16] Folgering, H. and Herwaorden, C. (1994) Exercise Limitation in Patients with Pulmonary Diseases. International Journal of Sports Medicine, 15, 107-111. http://dx.doi.org/10.1055/s-2007-1021029

[17] Martinez, J.A.B., Padua, A.I. and Terra, J. (2004) Dispnéia. Medicine, 37, 199-207. http://dx.doi.org/10.11606/issn.2176-7262.v37i3/4p199-207

[18] Hajiro, T., Nishimura, K., Jones, P.W., et al. (1999) Short and Simple Questionnaire to Measure Health Related Life Quality in Patients with Chronic Obstructive Pulmonary Disease. American Journal of Respiratory and Critical Care Medicine, 159, 1874-1878. http://dx.doi.org/10.1164/ajrccm.159.6.9807097

[19] Sanchez, F.F., Faganello, M.M., Tanni, S.E., et al. (2008) Relationship between Disease Severity and Life Quality in Patients with Chronic Obstructive Pulmonary Disease. Brazilian Journal of Medical and Biological Research, 41, 860865. http://dx.doi.org/10.1590/S0100-879X2008005000043 